\title{
A Strategy to Support Efficient Development and Use of Innovations in Personalized Medicine and Precision Medicine
}

\author{
Louis P. Garrison, Jr., PhD, and Adrian Towse, MPhil, MA
}

\section{SUMMARY}

This analysis addresses an important policy question: "What are potentially transformative strategies and methods to define and measure value at all levels of decision making that are aligned with personalized medicine/ precision medicine (PM/PrM)?" To approach this question, we build on our previous work with PM and recent work as members of ISPOR's Special Task Force on Value Assessment Frameworks. We focused on 3 key challenges: (1) the need for a broader concept of value for PM/PrM; (2) the development of appropriate concepts and methods for measuring and splitting the innovation reward for PM/PrM; and (3) the application of this expanded concept to align value assessment in the hierarchy of related decision contexts, from formulary inclusion and value-based pricing to shared patient-provider decision making.

Our working assumption was that the goal of policy affecting PM/PrM technologies should be to promote "dynamic efficiency"- the optimal development, adoption, and use of these technologies. For this purpose, we laid out 6 basic cross-cutting policy principles. First, rewards for innovation should be value-based and flexible over time and across indications as new evidence emerges. Second, the concept of value should be based on the microeconomic concept of willingness to pay and should include, at its core, the value of health gain-in terms of the length and quality of lifebut also going beyond this to include other uncertainty-related elements. Third, innovation rewards should be modifiable given new real-world evidence and knowledge. Fourth, the scientific information embedded in PM and PrM technologies is a global public good that should be financed globally through differential pricing. Fifth, reward systems need to recognize that the diagnostic technologies that make PM and PrM possible are technically "economic complements." Sixth, splitting the rewards among complementary inputs is arbitrary in a static sense but matters dynamically.

For PM/PrM value assessment, we argue for including the "value of knowing"; the need for indication-specific pricing; the importance of updating the economic evaluation and pricing over the product life cycle; addressing the new challenge posed by next-generation sequencing and high-cost combination therapies; and aligning value metrics across different decision contexts based on health benefit and patient-centered utility.

J Manag Care Spec Pharm. 2019;25(10):1082-87

Copyright $\odot 2019$, Academy of Managed Care Pharmacy. All rights reserved.

$\mathrm{I}$ $\mathrm{n}$ this analysis, we address an important policy question posed by the PhRMA Foundation-Personalized Medicine LCoalition Challenge Awards Request For Proposal: "What are potentially transformative strategies and methods aligned with personalized medicine/precision medicine (PM/PrM) to define and measure value at all levels of decision making?"

Our approach to this question builds on our previous work on personalized (or "stratified") medicine combined with our recent work as members of ISPOR's Special Task Force (STF) on Value Assessment Frameworks. ${ }^{1-8}$ We focus on addressing 3 key challenges that must be met to enhance the ability of the U.S. health care system to optimally incorporate PM/PrM. First, to support PM/PrM development and adoption, we need to use a broader concept of value that builds on the conventional cost-effectiveness ratio of the cost per quality-adjusted life-year (QALY). A second challenge is developing appropriate concepts and methods for measuring and splitting the innovation reward among the various complementary inputs used in the provision of PM and PrM to support efficient competition across technologies and diseases. Finally, the application of this expanded concept of value needs to be aligned in the hierarchy of several related decision contexts-from health plan selection, to inclusion in a health plan benefit package, down to the patient-centered, shared decision-making context.

For this discussion, we consider the terms "personalized," "stratified," and "precision" (PrM) as synonyms, recognizing that the PM concept is more population based, while PrM operates at the level of the individual, where multiple mutations may argue for a specific combination of medicines. ${ }^{9}$

With the 2003 sequencing of the human genome, many predicted or expected an immediate wave of medical breakthroughs; however, progress has been slow. The reasons for this are complex. The main factor is probably that the science itself has proven to be more complex than the optimists understood. But other factors, such as regulatory or reimbursement systems, are not set up to speed PM/PrM development. .,3,10 $^{2}$

Developing a transformative strategy that is implementable in the U.S. health care system is difficult given the cumulative complexity of our public-private system: There are many stakeholders invested in current rules and regulations at a multitude of policy margins. ${ }^{911}$ PM and PrM are late entrants to this environment as somewhat unanticipated add-ons to a system decades in the making, with a reward system for payment and reimbursement that is not designed to optimize the development and use of these new technologies. ${ }^{3,10}$

\section{Principles to Support a Strategy}

We assume the goal of policy changes affecting PM and PrM technologies should be to promote "dynamic efficiency"- the optimal development, adoption, and use of these technologies over time. Static efficiency decisions (i.e., about which ones to adopt given a fixed budget) need to be well aligned with other incentives to support this long-term objective. 


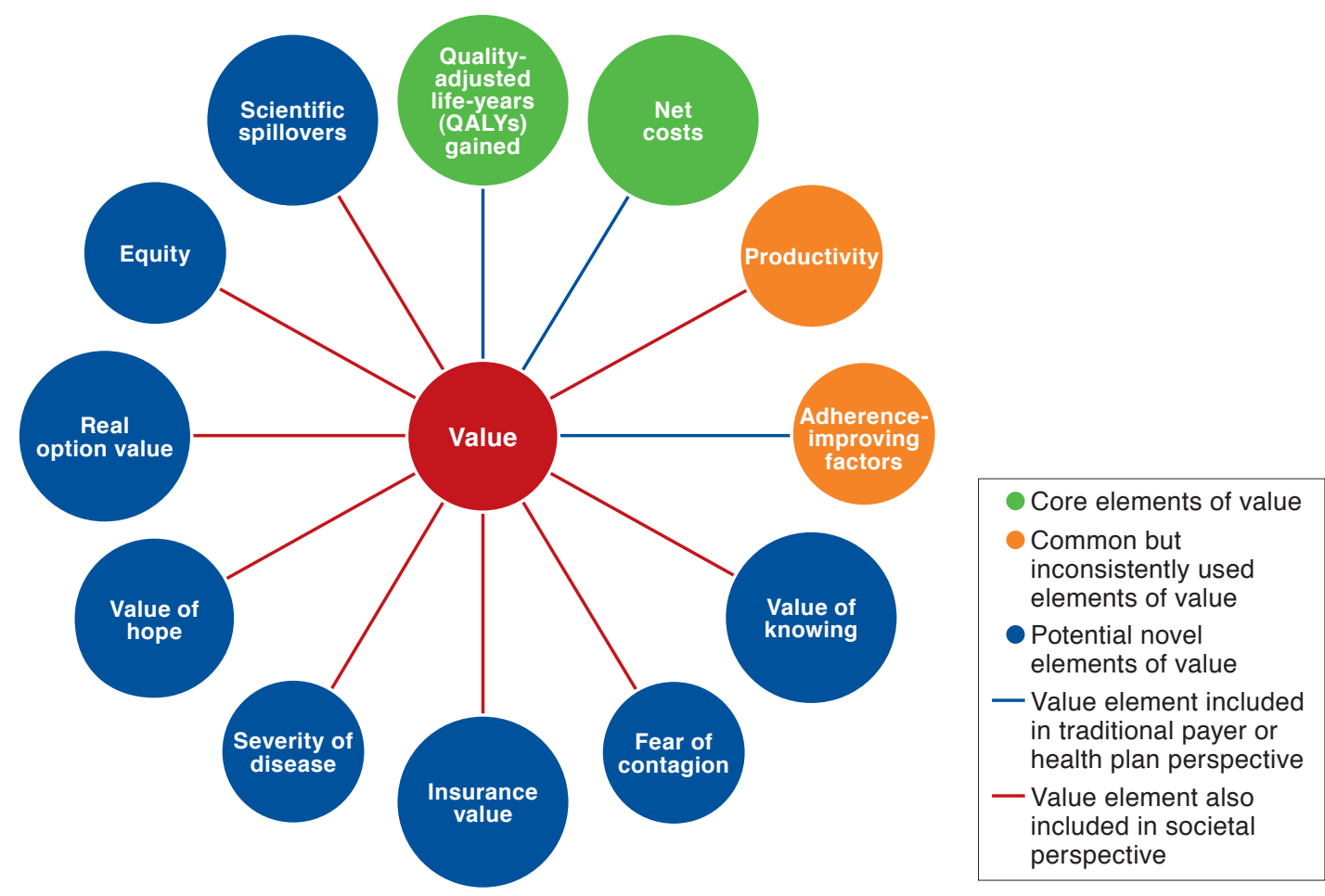

Adapted from: Lakdawalla DN, Doshi JA, Garrison LP Jr, Phelps CE, Basu A, Danzon PM. Defining elements of value in health care-a health economics approach: an ISPOR Special Task Force Report [3], p. 132.7 Reprinted with permission.

Supporting this goal requires following some basic crosscutting policy principles:

1. Rewards for innovation should be value based and flexible over time and across indications as new evidence emerges, with new technologies competing with old on a level playing field.

2. The concept of value should be based on the microeconomic theoretical concept of ex ante willingness to pay and should include, at its core, the value of health gain in terms of the length and quality of life. But it should also go beyond this to include other relevant elements that affect enrollee and patient well-being, including the value of knowing, which is particularly important in the application of $\mathrm{PM} / \mathrm{PrM}$.

3. For rewards to be flexible over time, they must be modifiable in light of new real-world evidence and emerging competing new technologies.

4. The scientific information embedded in PM and PrM technologies is a global public good. This means that globally everyone has a stake in supporting the required research and development and should contribute in proportion to their ability and willingness to pay.
5. Reward systems need to recognize that the diagnostic technologies that make PM and PrM possible are technically "economic complements" to the other inputs, including companion medicine or the prescribing provider.

6. Given the fundamental arbitrariness of splitting the rewards among inputs that are complementary in a static sense, reward systems for PM and PrM will need pragmatic rules that align dynamic stakeholder incentives across technologies and decision contexts.

\section{Defining Value for PM and PrM}

We follow the ISPOR STF report in defining value from a microeconomic perspective: "gross value' can be thought of what someone would be willing to pay for an economic good or intervention while the 'net value' subtracts the opportunity cost incurred to obtain that gross value." Valuations will vary across individuals, across indications for the same medicine, and dynamically over time. And value is difficult to measure in health care because of insurance. Cost-effectiveness analysis, including the pragmatic metric of the cost per QALY, helps to address these difficulties (within a budget or affordability constraint). 


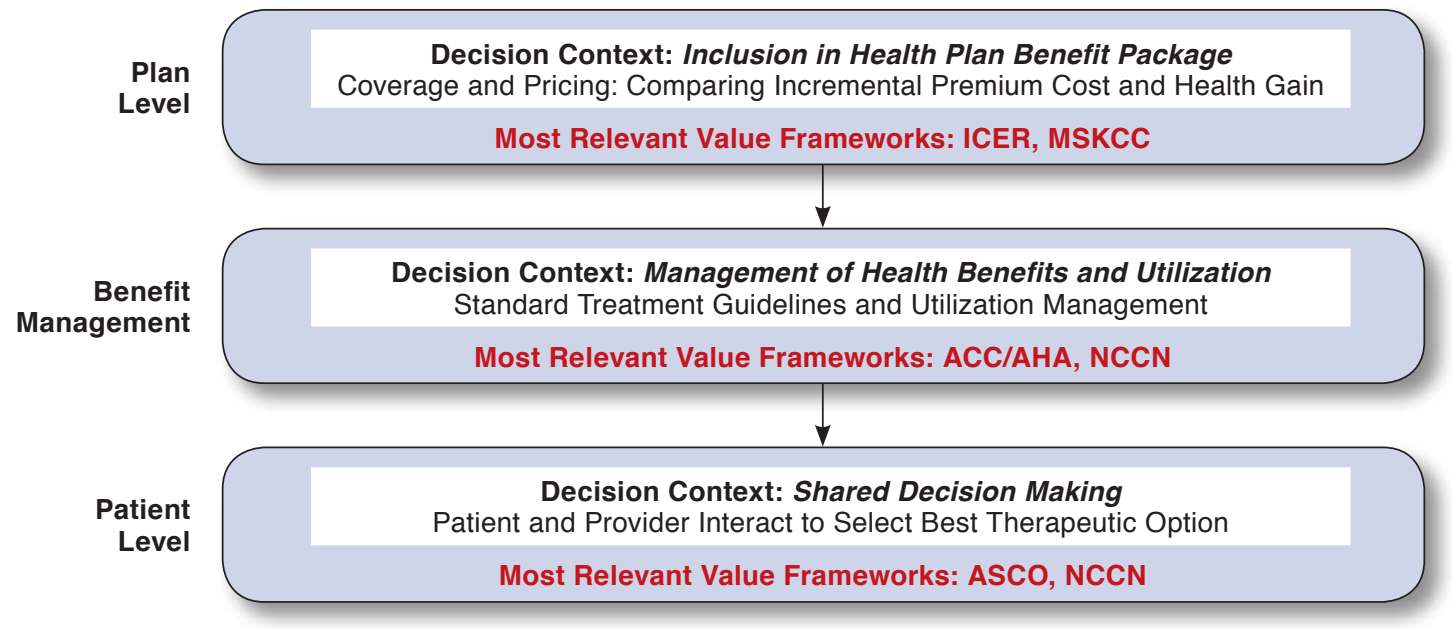

Source: Garrison LP Jr, Pauly MV, Willke RJ, Neumann PJ. An overview of value, perspective, and decision context-a health economics approach: an ISPOR Special Task Force Report [2], p. 127. ${ }^{8}$ Reprinted with permission.

ACC =American College of Cardiology; AHA=American Heart Association; ASCO=American Society of Clinical Oncology; ICER=Institute for Clinical and Economic Research; MSKCC = Memorial Sloan Kettering Cancer Center; NCCN = National Comprehensive Cancer Network.

\section{Expanding the Economic Concept of Value}

In a series of policy analyses, we have argued that patients and/or their agents (e.g., their payer) would be willing to pay more for the greater certainty of response, other things being equal, such as income and education. ${ }^{1-5,9}$ We have characterized this as the "value of knowing" for the patient. Figure 1 depicts an expanded concept of value as a "value flower," with each petal representing a different element. A number of these petals are related to uncertainty: value of knowing (from reducing uncertainty), insurance value (from protection for financial and health risk), value of hope (from cures), and real option value (from a richer development pipeline). An expanded concept of value should apply to all health technologies and is particularly relevant to the complementary diagnostic that supports PM/PrM. ${ }^{12}$

To incentivize and reward value creation in PM/PrM, we argue that enrollees (i.e., potential patients) should be willing to pay for the expected value generated by many of the elements in the value flower, subject to the budget or affordability constraint. ${ }^{10,13}$

\section{Rewarding Value by Indication: Indication-Specific Pricing} Given the variability in the treatment effects for a given medication, the value of PM or PrM technology would vary across individuals and among subpopulations, including those with different clinical indications such as metastatic versus early-stage cancer. In theory, supporting a level playing field across interventions would dictate varying the rewards by indication. ${ }^{14,15}$ In practice, this has been difficult to implement. However, since much of the buying is by a third party (i.e., the health plan) with confidential discounting, it would be feasible to give differential discounts by indication.

Implementing this requires an estimate of the value of use by indication. Arguably, the measurement of this distribution would not have to be perfect to be an improvement over the current situation. Indeed, an average price based on a plausible, assumed distribution would be workable, as Pearson et al. (2017) have called a "weighted average price". ${ }^{15}$ Chandra and Garthwaite (2017) have correctly pointed out that indicationspecific pricing would not necessarily reduce overall costs and could increase manufacturer's revenues for a given product. ${ }^{16}$ However, it could still be economically efficient if the price in the lowest value indication is proportionate to its relative value and still above short-run marginal cost and if patient access increases. ${ }^{17}$ A recent analysis suggests that implementation of indication-specific pricing in the United States may be feasible, and it is being pilot-tested by Express Scripts and CVS Caremark. ${ }^{15}$

\section{Updating and the Need for Real-World Evidence}

Given the objective of flexible, value-based pricing of health technologies, then coverage, prices, reimbursement, and even use will need to be adjusted over time-over the product life cycle. As a public good, real-world evidence will be undersupplied in free markets ${ }^{3}$ - hence, the need for patents and subsidies for generation of real-world evidence. 
Historically, payers have focused on assessing likely costeffectiveness and value at launch. Interestingly, the use of performance-based risk-sharing arrangements (PBRSAs) to address the uncertainties extant at launch can be interpreted as a means to generate needed real-world evidence. ${ }^{18}$ It is noteworthy that the United States-with its decentralized private insurance system of periodic drug price recontracting and the associated pharmacy and therapeutics committee processhas been much more open to changing prices and utilization management based on the latest information than some European countries, which rely more on PBRSAs.

\section{Complementarity and the Challenge for PM/PrM}

When a diagnostic test is used to target a medicine for a subpopulation of patients, the test and the medicine are economic complements. ${ }^{2,3}$ From the patient's point of view, value comes from use in combination. The patient is indifferent as to how that associated payment/reward is divided. In practice, given patent realities and reimbursement rules, most of the value has historically been captured by the drug. Oncotype DX, a test to predict breast cancer recurrence, was able to navigate these complexities to become one of the first-if not, the firstvalue-capturing predictive genetic tests. ${ }^{13,19}$

Reimbursement and legal rules may have been barriers to the uptake of PM. Our work as part of the UK Academy of Medical Sciences effort led to 2 key recommendations-\#14 and \#15-related to these barriers. ${ }^{3,20}$ From a static economic perspective, it is arbitrary how the value reward is divided among complementary inputs. But, from a dynamic perspective, it can affect the supply of future innovations, and we propose a pragmatic allocation to reward a PM diagnostic based on the additional value that it creates by avoiding adverse events among nonresponders.

\section{The New Challenge of Combination Treatment}

A parallel issue arises in the application of PrM using nextgeneration sequencing in oncology, where a combination of innovative medicines may be used based on the mutational signature of a cancer. ${ }^{21}$ How should the value-based reward be divided among the combined high-cost medicines? Often, via randomized controlled trials, we can get some sense of the marginal value contribution of adding a drug to create a combination. For example, the recent case of the addition of pertuzumab to trastuzumab for HER2+ metastatic breast cancer is illustrative of the potential complexity of operationalizing combination pricing. Median progression-free survival in the trastuzumab arm was 12.4 months versus 18.7 months in the arm when pertuzumab was added. ${ }^{22}$ Thus, there is a clear idea of the marginal health gain as the implied QALYs gained from the extra 6 months. However, there is a perverse consequence related to the per-vial reimbursement system. Since patients typically receive treatment until progression, the sales of trastuzumab will also increase with addition of pertuzumab. Unlike the instance where the same company sells both drugs, if a second company had marketed pertuzumab, it would not capture the full marginal value under current reimbursement practices and would thus be discouraged from development.

These complexities can, however, be multiplied in the application of PrM. Consider a circumstance where a PrM-based 2-drug combination is tried but without a previous trial with only 1 of the 2 agents. Then, it is impossible to determine the marginal value. Historically, this has been rare given the regulated sequence of drug development, but with the advent of PrM via multigene panel tests, the door has been opened wider for physicians to experiment off-label with high-cost combinations not in randomized controlled trials. In late 2017, the U.S. Food and Drug Administration approved FoundationOne CDx, a broad companion oncologic diagnostic for 324 genes potentially related to solid tumors and immunotherapy. ${ }^{23}$ The test costs about $\$ 5,800$, and Medicare has already approved its use in all advanced solid tumors and cancer.

In principle, we could try (using observational comparison) to estimate the marginal value (beyond the now fixed cost of testing) in the subgroup of patients receiving the drug combination and divide that among the component drugs. But, in the absence of indication-specific pricing adjustments, this will be challenging if the drugs are each high cost. An important research topic is, however, to explore if rules that split the reward differentially could incentivize other desirable developer behavior, for example, investments in trials for other indications or with longer follow-up.

\section{Alignment in the Decision Context Hierarchy}

The ISPOR STF attempted to make it clear that one of the reasons for confusion about value frameworks is that they have been developed to support different decisions. ${ }^{8}$ Figure 2 depicts a hierarchy or-perhaps better-a cascade of decision contexts. $^{8}$ At the top level, the Institute for Clinical and Economic Review's framework based on the cost-per-QALY metric (to be adjusted for other factors) aims to support health plan decisions about inclusion in the formulary and pricing negotiations. At the next level down of benefit management, after a drug is in the formulary, there is a need for treatment guidelines, pathways, and utilization management (i.e., prior authorization and step edits) to promote "appropriate" use. We support the recommendation by the American College of Cardiology/American Heart Association to use conventional cost-effectiveness analysis in developing treatment guidelines, but this should expand the concept of value to the STF's "augmented" cost-effectiveness analysis. ${ }^{7}$ This middle level is about the stewardship of society's scarce resources and puts a useful constraint on the providerpatient interaction.

The lowest level in this hierarchy is the all-important patientcentric level: It is where the provider (as agent) advises the patient. Value frameworks such as those of the American Society of Clinical Oncology (ASCO) and National Comprehensive Cancer Network aim to support decision making with a concept of clinical value. The simplest and most straightforward way to 
align this with the levels previously mentioned is to use QALY or an expanded QALY as the indicator of clinical benefit. Both ASCO and health economists often call this "net health benefit" (NHB), presenting the QALY-based, patient-centric core to align the NHB measure across all 3 levels. ${ }^{25}$

There are, however, 2 other important contexts not addressed: (1) when enrollees (potential patients) choose among health plans and (2) regulatory benefit-risk assessment. It is possible that value-based health plan formularies could compete by communicating whether and how they use the cost-effectiveness analysis metrics. ${ }^{24}$ At the regulatory level, systematic and quantitative benefit-risk analyses could use QALY to assess benefit-risk balance, while using value of information analysis to assess further data collection. ${ }^{25-27}$

\section{Methods to Support a Strategy}

We support the recommendation of the ISPOR STF to build on the cost-per-QALY concept. ${ }^{5}$ PM/PRM demands a broader concept of value, given its effect on uncertainty. There are potential approaches for valuing and aggregating additional values for decision making: (1) including more attributes in the health state descriptions for health state utility estimation; (2) moving toward a "net monetary benefit" comparison by monetizing the QALY6; (3) using multicriteria decision analysis to estimate weights through a group process ${ }^{24}$; and (4) using other structured deliberative processes to review the evidence and vote. ${ }^{6}$ We support the STF's recommendations that more research is needed on each of these approaches.

\section{Conclusions}

In preparing this policy analysis, we have attempted to weave together several threads of our previous collaborative research on value in PM/PrM. Our proposed strategy for transformative change has 5 key elements: (1) broadening the concept of value to better identify and reward the contribution of PM/ PrM diagnostics; (2) implementing indication-specific pricing in the United States; (3) investing more in the generation of real-world evidence for PM/PrM; (4) reforming how we measure and reward value creation in PM/PrM; and (5) aligning value metrics across different decision contexts built on health benefit based on patient-centered utility.

\section{Authors}

LOUIS P. GARRISON, Jr., PhD, The Comparative Health Outcomes, Policy, and Economics (CHOICE) Institute, University of Washington, Seattle, and ADRIAN TOWSE, MPhil, MA, The Office of Health Economics, London, United Kingdom.

AUTHOR CORRESPONDENCE: Louis P. Garrison, Jr., PhD, Professor Emeritus, The Comparative Health Outcomes, Policy, and Economics (CHOICE) Institute, Department of Pharmacy, University of Washington, Seattle, WA 98195-7630. Tel.: 206.427.0798;

E-mail: lgarrisn@uw.edu.

\section{DISCLOSURES}

No previous outside funding supported this policy analysis. The authors have nothing to disclose.

The authors are grateful to have received first place in the PhRMA Foundation-Personalized Medicine Coalition 2018 Value Assessment Challenge Award for this work.

\section{REFERENCES}

1. Faulkner E, Annemans L, Garrison L, et al. Challenges in the development and reimbursement of personalized medicine-payer and manufacturer perspectives and implications for health economics and outcomes research: a report of the ISPOR Personalized Medicine Special Interest Group. Value Health. 2012;15(8):1162-71

2. Towse A, Garrison LP Jr. Economic incentives for evidence generation: promoting an efficient path to personalized medicine. Value Health. 2013;16(6 Suppl):S39-43

3. Garrison LP, Towse A. Economics of personalized medicine: pricing and reimbursement policies as a potential barrier to development and adoption In: Culyer T, ed. Encyclopedia of Health Economics. Oxford: Elsevier; 2014.

4. Garrison L, Mestre-Ferrandiz J, Zamora B. The value of knowing and knowing the value: improving the health technology assessment of complementary diagnostics. White paper. Office of Health Economics and EPEMED. July 2016. Available at: https://www.ohe.org/sites/default/files/ WP_EpemedOHE_final.pdf. Accessed September 1, 2019.

5. Garrison LP, Towse A. Value-based pricing and reimbursement in personalised healthcare: introduction to the basic health economics. J Pers Med. September 4, 2017 [Epub]. Available at: https://www.ncbi.nlm.nih.gov/pmc/ articles/PMC5618156/. Accessed September 1, 2019.

6. Garrison LP Jr, Neumann PJ, Willke RJ, et al. A health economics approach to us value assessment frameworks-summary and recommendations of the ISPOR Special Task Force Report [7]. Value Health. 2018;21(2):161-65.

7. Lakdawalla DN, Doshi JA, Garrison LP Jr, Phelps CE, Basu A, Danzon PM. Defining elements of value in health care-a health economics approach: an ISPOR Special Task Force Report [3]. Value Health. 2018;21(2):131-39.

8. Garrison LP Jr, Pauly MV, Willke RJ, Neumann PJ. An overview of value, perspective, and decision context-a health economics approach: an ISPOR Special Task Force Report [2]. Value Health. 2018;21(2):124-30.

9. Personalized Medicine Coalition. The personalized medicine report: opportunities, challenges, and the future. 2017. Available at: http://www. personalizedmedicinecoalition.org/Userfiles/PMC-Corporate/file/ThePersonalized-Medicine-Reportl.pdf. Accessed September 1, 2019.

10. Garrison LP, Austin MJF. Linking pharmacogenetics-based diagnostics and pharmaceuticals for personalized medicine: scientific and economic challenges. Health Aff. 2006;25(5):1281-90. Available at: https://www.healthaffairs. org/doi/pdf/10.1377/hlthaff.25.5.1281. Accessed September 1, 2019.

11. U.S. Department of Health and Human Services. HHS Blueprint of Lower Drug Prices and Reduce Out-of-Pocket Costs. Fed Reg. 2018;83(95):22692-700. Available at: https://www.federalregister.gov/documents/2018/05/16/2018-10435/hhs-blueprint-to-lower-drug-prices-andreduce-out-of-pocket-costs. Accessed September 1, 2019.

12. Garrison LP, Kamal-Bahl, S, Towse, A. Toward a broader concept of value: identifying and defining elements for an expanded cost-effectiveness analysis. Value Health. 2017;20(2):213-16.

13. Garau M, Towse A, Garrison L, Housman L, Ossa D. Can and should value-based pricing be applied to molecular diagnostics? Per Med. 2013;10(1):61-72.

14. Garrison LP. Rewarding value creation to promote innovation in the oncology: the importance of considering the global product life cycle. Oncologist. 2010;15(Suppl 1):49-57. 
15. Pearson SD, Dreitlein WB, Henshall C, Towse A. Indication-specific pricing of pharmaceuticals in the U.S. healthcare system. J Comp Eff Res. 2017;6(5):397-404.

16. Chandra A, Garthwaite C. The economics of indication-based drug pricing. N Engl J Med. 2017;377(2):103-06.

17. Cole A, Towse A, Lorgelly P, Sullivan R. Economics of innovative payment models compared with single pricing of pharmaceuticals. Office of Health Economics Research Paper 18/04. July 2018. Available at: https:// www.ohe.org/publications/economics-innovative-payment-models-compared-single-pricing-pharmaceuticals-0\#. Accessed September 1, 2019.

18. Garrison LP Jr, Towse A, Briggs A, et al. Performance-based risk-sharing arrangements—-good practices for design, implementation, and evaluation: report of the ISPOR Good Practices for Performance-Based Risk-Sharing Arrangements Task Force. Value Health. 2013;16(5):703-19.

19. Towse A, Ossa D, Veenstra D, Carlson J, Garrison L. Understanding the economic value of molecular diagnostic tests: case studies and lessons learned. J Pers Med. 2013;3(4):288-305.

20. The Academy of Medical Sciences. Realizing the potential of stratified medicine. July 2013. Available at: https://acmedsci.ac.uk/viewFile/ 5le915f9f09fb.pdf. Accessed September 1, 2019.
21. Phillips KA, Deverka PA, Marshall DA, et al. Methodological issues in assessing the economic value of next-generation sequencing tests: many challenges and not enough solutions. Value Health. 2018;21(9):1033-42.

22. Swain SM, Kim SB, Cortés J, et al. Pertuzumab, trastuzumab, and docetaxel for HER2-positive metastatic breast cancer (CLEOPATRA study): overall survival results from a randomised, double-blind, placebo-controlled, phase 3 study. Lancet Oncol. 2013;14(6):461-71.

23. Phillips KA, Trosman JR, Deverka PA, et al. Insurance coverage for genomic tests. Science. 2018;360(6386):278-79.

24. Danzon PM, Drummond MF, Towse A, Pauly MV. Objectives, budgets, thresholds, and opportunity costs - a health economics approach: an ISPOR Special Task Force Report [4]. Value Health. 2018;21(2):140-45.

25. Garrison LP, Towse A, Bresnahan B. Assessing a structured, quantitative health outcomes approach to drug risk-benefit analysis. Health Aff (Millwood). 2007;26(3):684-95.

26. Garrison LP. Regulatory benefit-risk assessment and comparative effectiveness research: strangers, bedfellows or strange bedfellows? Pharmacoeconomics. 2010;28(10):855-65.

27. Phelps CE, Lakdawalla DN, Basu A, Drummond MF, Towse A, Danzon PM. Approaches to aggregation and decision making - a health economics approach: an ISPOR Special Task Force Report [5]. Value Health. 2018;21(2):146-54. 